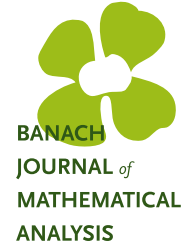

Banach J. Math. Anal. 10 (2016), no. 4, 686-702

http://dx.doi.org/10.1215/17358787-3649854

ISSN: $1735-8787$ (electronic)

http://projecteuclid.org/bjma

ANALYSIS

\title{
IDEAL STRUCTURES IN VECTOR-VALUED POLYNOMIAL SPACES
}

\author{
VERÓNICA DIMANT, ${ }^{1}$ SILVIA LASSALLE, ${ }^{1}$ and ÁNGELES PRIETO ${ }^{2 *}$ \\ Communicated by D. Werner
}

\begin{abstract}
This paper is concerned with the study of geometric structures in spaces of polynomials. More precisely, we discuss for $E$ and $F$ Banach spaces, whether the class of $n$-homogeneous polynomials, $\mathcal{P}_{w}\left({ }^{n} E, F\right)$, which are weakly continuous on bounded sets, is an HB-subspace or an $M(1, C)$-ideal in the space of continuous $n$-homogeneous polynomials, $\mathcal{P}\left({ }^{n} E, F\right)$. We establish sufficient conditions under which the problem can be positively solved. Some examples are given. We also study when some ideal structures pass from $\mathcal{P}_{w}\left({ }^{n} E, F\right)$ as an ideal in $\mathcal{P}\left({ }^{n} E, F\right)$ to the range space $F$ as an ideal in its bidual $F^{* *}$.
\end{abstract}

\section{INTRODUCTION}

Let $X$ be a (real or complex) Banach space, and let $J$ be a closed subspace of $X$. According to the Hahn-Banach theorem, every continuous linear functional $g \in J^{*}$ has an extension $f \in X^{*}$ with the same norm. A long-standing problem is to determine when every functional on $J$ has a unique norm-preserving extension to $X$. This question is closely related to geometric properties of both spaces which, in many cases, imply the existence of a norm 1 projection on $X^{*}$ whose kernel is $J^{\perp}:=\left\{x^{*} \in X^{*}: x^{*}(y)=0\right.$, for all $\left.y \in J\right\}$, the annihilator of $J$. When there exists such a projection, $J$ is said to be an ideal in $X$. A canonical example of this fact is that $X$ is always an ideal in its bidual $X^{* *}$.

The notion of $M$-ideal, introduced by Alfsen and Effros and widely studied by Harmand, Werner, and Werner in [18], is one of these geometric properties

Copyright 2016 by the Tusi Mathematical Research Group.

Received Dec. 3, 2015; Accepted Dec. 22, 2015.

${ }^{*}$ Corresponding author.

2010 Mathematics Subject Classification. Primary 46G25; Secondary 47H60, 46B04, 47L22.

Keywords. HB-subspaces, homogeneous polynomials, weakly continuous on bounded sets polynomials. 


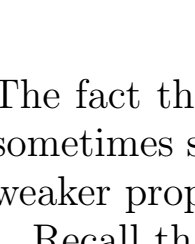

Banach J. Math. Anal. 10 (2016), no. 4, 686-702

http://dx.doi.org/10.1215/17358787-3649854

ISSN: $1735-8787$ (electronic)

http://projecteuclid.org/bjma

ANALYSIS

\title{
IDEAL STRUCTURES IN VECTOR-VALUED POLYNOMIAL SPACES
}

\author{
VERÓNICA DIMANT, ${ }^{1}$ SILVIA LASSALLE, ${ }^{1}$ and ÁNGELES PRIETO ${ }^{2 *}$ \\ Communicated by D. Werner
}

\begin{abstract}
This paper is concerned with the study of geometric structures in spaces of polynomials. More precisely, we discuss for $E$ and $F$ Banach spaces, whether the class of $n$-homogeneous polynomials, $\mathcal{P}_{w}\left({ }^{n} E, F\right)$, which are weakly continuous on bounded sets, is an HB-subspace or an $M(1, C)$-ideal in the space of continuous $n$-homogeneous polynomials, $\mathcal{P}\left({ }^{n} E, F\right)$. We establish sufficient conditions under which the problem can be positively solved. Some examples are given. We also study when some ideal structures pass from $\mathcal{P}_{w}\left({ }^{n} E, F\right)$ as an ideal in $\mathcal{P}\left({ }^{n} E, F\right)$ to the range space $F$ as an ideal in its bidual $F^{* *}$.
\end{abstract}

\section{INTRODUCTION}

Let $X$ be a (real or complex) Banach space, and let $J$ be a closed subspace of $X$. According to the Hahn-Banach theorem, every continuous linear functional $g \in J^{*}$ has an extension $f \in X^{*}$ with the same norm. A long-standing problem is to determine when every functional on $J$ has a unique norm-preserving extension to $X$. This question is closely related to geometric properties of both spaces which, in many cases, imply the existence of a norm 1 projection on $X^{*}$ whose kernel is $J^{\perp}:=\left\{x^{*} \in X^{*}: x^{*}(y)=0\right.$, for all $\left.y \in J\right\}$, the annihilator of $J$. When there exists such a projection, $J$ is said to be an ideal in $X$. A canonical example of this fact is that $X$ is always an ideal in its bidual $X^{* *}$.

The notion of $M$-ideal, introduced by Alfsen and Effros and widely studied by Harmand, Werner, and Werner in [18], is one of these geometric properties

Copyright 2016 by the Tusi Mathematical Research Group.

Received Dec. 3, 2015; Accepted Dec. 22, 2015.

${ }^{*}$ Corresponding author.

2010 Mathematics Subject Classification. Primary 46G25; Secondary 47H60, 46B04, 47L22.

Keywords. HB-subspaces, homogeneous polynomials, weakly continuous on bounded sets polynomials. 


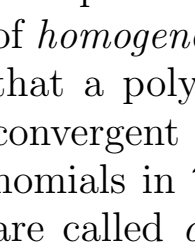

Banach J. Math. Anal. 10 (2016), no. 4, 686-702

http://dx.doi.org/10.1215/17358787-3649854

ISSN: $1735-8787$ (electronic)

http://projecteuclid.org/bjma

ANALYSIS

\title{
IDEAL STRUCTURES IN VECTOR-VALUED POLYNOMIAL SPACES
}

\author{
VERÓNICA DIMANT, ${ }^{1}$ SILVIA LASSALLE, ${ }^{1}$ and ÁNGELES PRIETO ${ }^{2 *}$ \\ Communicated by D. Werner
}

\begin{abstract}
This paper is concerned with the study of geometric structures in spaces of polynomials. More precisely, we discuss for $E$ and $F$ Banach spaces, whether the class of $n$-homogeneous polynomials, $\mathcal{P}_{w}\left({ }^{n} E, F\right)$, which are weakly continuous on bounded sets, is an HB-subspace or an $M(1, C)$-ideal in the space of continuous $n$-homogeneous polynomials, $\mathcal{P}\left({ }^{n} E, F\right)$. We establish sufficient conditions under which the problem can be positively solved. Some examples are given. We also study when some ideal structures pass from $\mathcal{P}_{w}\left({ }^{n} E, F\right)$ as an ideal in $\mathcal{P}\left({ }^{n} E, F\right)$ to the range space $F$ as an ideal in its bidual $F^{* *}$.
\end{abstract}

\section{INTRODUCTION}

Let $X$ be a (real or complex) Banach space, and let $J$ be a closed subspace of $X$. According to the Hahn-Banach theorem, every continuous linear functional $g \in J^{*}$ has an extension $f \in X^{*}$ with the same norm. A long-standing problem is to determine when every functional on $J$ has a unique norm-preserving extension to $X$. This question is closely related to geometric properties of both spaces which, in many cases, imply the existence of a norm 1 projection on $X^{*}$ whose kernel is $J^{\perp}:=\left\{x^{*} \in X^{*}: x^{*}(y)=0\right.$, for all $\left.y \in J\right\}$, the annihilator of $J$. When there exists such a projection, $J$ is said to be an ideal in $X$. A canonical example of this fact is that $X$ is always an ideal in its bidual $X^{* *}$.

The notion of $M$-ideal, introduced by Alfsen and Effros and widely studied by Harmand, Werner, and Werner in [18], is one of these geometric properties

Copyright 2016 by the Tusi Mathematical Research Group.

Received Dec. 3, 2015; Accepted Dec. 22, 2015.

${ }^{*}$ Corresponding author.

2010 Mathematics Subject Classification. Primary 46G25; Secondary 47H60, 46B04, 47L22.

Keywords. HB-subspaces, homogeneous polynomials, weakly continuous on bounded sets polynomials. 


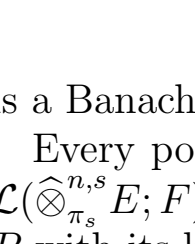

Banach J. Math. Anal. 10 (2016), no. 4, 686-702

http://dx.doi.org/10.1215/17358787-3649854

ISSN: $1735-8787$ (electronic)

http://projecteuclid.org/bjma

ANALYSIS

\title{
IDEAL STRUCTURES IN VECTOR-VALUED POLYNOMIAL SPACES
}

\author{
VERÓNICA DIMANT, ${ }^{1}$ SILVIA LASSALLE, ${ }^{1}$ and ÁNGELES PRIETO ${ }^{2 *}$ \\ Communicated by D. Werner
}

\begin{abstract}
This paper is concerned with the study of geometric structures in spaces of polynomials. More precisely, we discuss for $E$ and $F$ Banach spaces, whether the class of $n$-homogeneous polynomials, $\mathcal{P}_{w}\left({ }^{n} E, F\right)$, which are weakly continuous on bounded sets, is an HB-subspace or an $M(1, C)$-ideal in the space of continuous $n$-homogeneous polynomials, $\mathcal{P}\left({ }^{n} E, F\right)$. We establish sufficient conditions under which the problem can be positively solved. Some examples are given. We also study when some ideal structures pass from $\mathcal{P}_{w}\left({ }^{n} E, F\right)$ as an ideal in $\mathcal{P}\left({ }^{n} E, F\right)$ to the range space $F$ as an ideal in its bidual $F^{* *}$.
\end{abstract}

\section{INTRODUCTION}

Let $X$ be a (real or complex) Banach space, and let $J$ be a closed subspace of $X$. According to the Hahn-Banach theorem, every continuous linear functional $g \in J^{*}$ has an extension $f \in X^{*}$ with the same norm. A long-standing problem is to determine when every functional on $J$ has a unique norm-preserving extension to $X$. This question is closely related to geometric properties of both spaces which, in many cases, imply the existence of a norm 1 projection on $X^{*}$ whose kernel is $J^{\perp}:=\left\{x^{*} \in X^{*}: x^{*}(y)=0\right.$, for all $\left.y \in J\right\}$, the annihilator of $J$. When there exists such a projection, $J$ is said to be an ideal in $X$. A canonical example of this fact is that $X$ is always an ideal in its bidual $X^{* *}$.

The notion of $M$-ideal, introduced by Alfsen and Effros and widely studied by Harmand, Werner, and Werner in [18], is one of these geometric properties

Copyright 2016 by the Tusi Mathematical Research Group.

Received Dec. 3, 2015; Accepted Dec. 22, 2015.

${ }^{*}$ Corresponding author.

2010 Mathematics Subject Classification. Primary 46G25; Secondary 47H60, 46B04, 47L22.

Keywords. HB-subspaces, homogeneous polynomials, weakly continuous on bounded sets polynomials. 


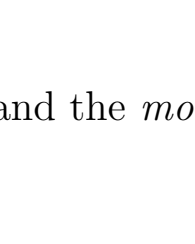

Banach J. Math. Anal. 10 (2016), no. 4, 686-702

http://dx.doi.org/10.1215/17358787-3649854

ISSN: $1735-8787$ (electronic)

http://projecteuclid.org/bjma

ANALYSIS

\title{
IDEAL STRUCTURES IN VECTOR-VALUED POLYNOMIAL SPACES
}

\author{
VERÓNICA DIMANT, ${ }^{1}$ SILVIA LASSALLE, ${ }^{1}$ and ÁNGELES PRIETO ${ }^{2 *}$ \\ Communicated by D. Werner
}

\begin{abstract}
This paper is concerned with the study of geometric structures in spaces of polynomials. More precisely, we discuss for $E$ and $F$ Banach spaces, whether the class of $n$-homogeneous polynomials, $\mathcal{P}_{w}\left({ }^{n} E, F\right)$, which are weakly continuous on bounded sets, is an HB-subspace or an $M(1, C)$-ideal in the space of continuous $n$-homogeneous polynomials, $\mathcal{P}\left({ }^{n} E, F\right)$. We establish sufficient conditions under which the problem can be positively solved. Some examples are given. We also study when some ideal structures pass from $\mathcal{P}_{w}\left({ }^{n} E, F\right)$ as an ideal in $\mathcal{P}\left({ }^{n} E, F\right)$ to the range space $F$ as an ideal in its bidual $F^{* *}$.
\end{abstract}

\section{INTRODUCTION}

Let $X$ be a (real or complex) Banach space, and let $J$ be a closed subspace of $X$. According to the Hahn-Banach theorem, every continuous linear functional $g \in J^{*}$ has an extension $f \in X^{*}$ with the same norm. A long-standing problem is to determine when every functional on $J$ has a unique norm-preserving extension to $X$. This question is closely related to geometric properties of both spaces which, in many cases, imply the existence of a norm 1 projection on $X^{*}$ whose kernel is $J^{\perp}:=\left\{x^{*} \in X^{*}: x^{*}(y)=0\right.$, for all $\left.y \in J\right\}$, the annihilator of $J$. When there exists such a projection, $J$ is said to be an ideal in $X$. A canonical example of this fact is that $X$ is always an ideal in its bidual $X^{* *}$.

The notion of $M$-ideal, introduced by Alfsen and Effros and widely studied by Harmand, Werner, and Werner in [18], is one of these geometric properties

Copyright 2016 by the Tusi Mathematical Research Group.

Received Dec. 3, 2015; Accepted Dec. 22, 2015.

${ }^{*}$ Corresponding author.

2010 Mathematics Subject Classification. Primary 46G25; Secondary 47H60, 46B04, 47L22.

Keywords. HB-subspaces, homogeneous polynomials, weakly continuous on bounded sets polynomials. 


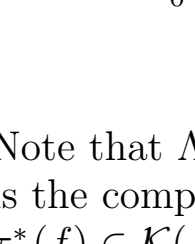

Banach J. Math. Anal. 10 (2016), no. 4, 686-702

http://dx.doi.org/10.1215/17358787-3649854

ISSN: $1735-8787$ (electronic)

http://projecteuclid.org/bjma

ANALYSIS

\title{
IDEAL STRUCTURES IN VECTOR-VALUED POLYNOMIAL SPACES
}

\author{
VERÓNICA DIMANT, ${ }^{1}$ SILVIA LASSALLE, ${ }^{1}$ and ÁNGELES PRIETO ${ }^{2 *}$ \\ Communicated by D. Werner
}

\begin{abstract}
This paper is concerned with the study of geometric structures in spaces of polynomials. More precisely, we discuss for $E$ and $F$ Banach spaces, whether the class of $n$-homogeneous polynomials, $\mathcal{P}_{w}\left({ }^{n} E, F\right)$, which are weakly continuous on bounded sets, is an HB-subspace or an $M(1, C)$-ideal in the space of continuous $n$-homogeneous polynomials, $\mathcal{P}\left({ }^{n} E, F\right)$. We establish sufficient conditions under which the problem can be positively solved. Some examples are given. We also study when some ideal structures pass from $\mathcal{P}_{w}\left({ }^{n} E, F\right)$ as an ideal in $\mathcal{P}\left({ }^{n} E, F\right)$ to the range space $F$ as an ideal in its bidual $F^{* *}$.
\end{abstract}

\section{INTRODUCTION}

Let $X$ be a (real or complex) Banach space, and let $J$ be a closed subspace of $X$. According to the Hahn-Banach theorem, every continuous linear functional $g \in J^{*}$ has an extension $f \in X^{*}$ with the same norm. A long-standing problem is to determine when every functional on $J$ has a unique norm-preserving extension to $X$. This question is closely related to geometric properties of both spaces which, in many cases, imply the existence of a norm 1 projection on $X^{*}$ whose kernel is $J^{\perp}:=\left\{x^{*} \in X^{*}: x^{*}(y)=0\right.$, for all $\left.y \in J\right\}$, the annihilator of $J$. When there exists such a projection, $J$ is said to be an ideal in $X$. A canonical example of this fact is that $X$ is always an ideal in its bidual $X^{* *}$.

The notion of $M$-ideal, introduced by Alfsen and Effros and widely studied by Harmand, Werner, and Werner in [18], is one of these geometric properties

Copyright 2016 by the Tusi Mathematical Research Group.

Received Dec. 3, 2015; Accepted Dec. 22, 2015.

${ }^{*}$ Corresponding author.

2010 Mathematics Subject Classification. Primary 46G25; Secondary 47H60, 46B04, 47L22.

Keywords. HB-subspaces, homogeneous polynomials, weakly continuous on bounded sets polynomials. 


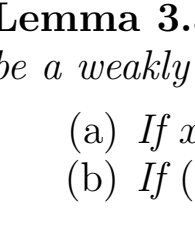

Banach J. Math. Anal. 10 (2016), no. 4, 686-702

http://dx.doi.org/10.1215/17358787-3649854

ISSN: $1735-8787$ (electronic)

http://projecteuclid.org/bjma

ANALYSIS

\title{
IDEAL STRUCTURES IN VECTOR-VALUED POLYNOMIAL SPACES
}

\author{
VERÓNICA DIMANT, ${ }^{1}$ SILVIA LASSALLE, ${ }^{1}$ and ÁNGELES PRIETO ${ }^{2 *}$ \\ Communicated by D. Werner
}

\begin{abstract}
This paper is concerned with the study of geometric structures in spaces of polynomials. More precisely, we discuss for $E$ and $F$ Banach spaces, whether the class of $n$-homogeneous polynomials, $\mathcal{P}_{w}\left({ }^{n} E, F\right)$, which are weakly continuous on bounded sets, is an HB-subspace or an $M(1, C)$-ideal in the space of continuous $n$-homogeneous polynomials, $\mathcal{P}\left({ }^{n} E, F\right)$. We establish sufficient conditions under which the problem can be positively solved. Some examples are given. We also study when some ideal structures pass from $\mathcal{P}_{w}\left({ }^{n} E, F\right)$ as an ideal in $\mathcal{P}\left({ }^{n} E, F\right)$ to the range space $F$ as an ideal in its bidual $F^{* *}$.
\end{abstract}

\section{INTRODUCTION}

Let $X$ be a (real or complex) Banach space, and let $J$ be a closed subspace of $X$. According to the Hahn-Banach theorem, every continuous linear functional $g \in J^{*}$ has an extension $f \in X^{*}$ with the same norm. A long-standing problem is to determine when every functional on $J$ has a unique norm-preserving extension to $X$. This question is closely related to geometric properties of both spaces which, in many cases, imply the existence of a norm 1 projection on $X^{*}$ whose kernel is $J^{\perp}:=\left\{x^{*} \in X^{*}: x^{*}(y)=0\right.$, for all $\left.y \in J\right\}$, the annihilator of $J$. When there exists such a projection, $J$ is said to be an ideal in $X$. A canonical example of this fact is that $X$ is always an ideal in its bidual $X^{* *}$.

The notion of $M$-ideal, introduced by Alfsen and Effros and widely studied by Harmand, Werner, and Werner in [18], is one of these geometric properties

Copyright 2016 by the Tusi Mathematical Research Group.

Received Dec. 3, 2015; Accepted Dec. 22, 2015.

${ }^{*}$ Corresponding author.

2010 Mathematics Subject Classification. Primary 46G25; Secondary 47H60, 46B04, 47L22.

Keywords. HB-subspaces, homogeneous polynomials, weakly continuous on bounded sets polynomials. 


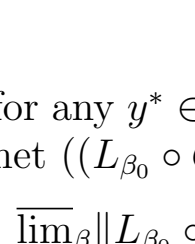

Banach J. Math. Anal. 10 (2016), no. 4, 686-702

http://dx.doi.org/10.1215/17358787-3649854

ISSN: $1735-8787$ (electronic)

http://projecteuclid.org/bjma

ANALYSIS

\title{
IDEAL STRUCTURES IN VECTOR-VALUED POLYNOMIAL SPACES
}

\author{
VERÓNICA DIMANT, ${ }^{1}$ SILVIA LASSALLE, ${ }^{1}$ and ÁNGELES PRIETO ${ }^{2 *}$ \\ Communicated by D. Werner
}

\begin{abstract}
This paper is concerned with the study of geometric structures in spaces of polynomials. More precisely, we discuss for $E$ and $F$ Banach spaces, whether the class of $n$-homogeneous polynomials, $\mathcal{P}_{w}\left({ }^{n} E, F\right)$, which are weakly continuous on bounded sets, is an HB-subspace or an $M(1, C)$-ideal in the space of continuous $n$-homogeneous polynomials, $\mathcal{P}\left({ }^{n} E, F\right)$. We establish sufficient conditions under which the problem can be positively solved. Some examples are given. We also study when some ideal structures pass from $\mathcal{P}_{w}\left({ }^{n} E, F\right)$ as an ideal in $\mathcal{P}\left({ }^{n} E, F\right)$ to the range space $F$ as an ideal in its bidual $F^{* *}$.
\end{abstract}

\section{INTRODUCTION}

Let $X$ be a (real or complex) Banach space, and let $J$ be a closed subspace of $X$. According to the Hahn-Banach theorem, every continuous linear functional $g \in J^{*}$ has an extension $f \in X^{*}$ with the same norm. A long-standing problem is to determine when every functional on $J$ has a unique norm-preserving extension to $X$. This question is closely related to geometric properties of both spaces which, in many cases, imply the existence of a norm 1 projection on $X^{*}$ whose kernel is $J^{\perp}:=\left\{x^{*} \in X^{*}: x^{*}(y)=0\right.$, for all $\left.y \in J\right\}$, the annihilator of $J$. When there exists such a projection, $J$ is said to be an ideal in $X$. A canonical example of this fact is that $X$ is always an ideal in its bidual $X^{* *}$.

The notion of $M$-ideal, introduced by Alfsen and Effros and widely studied by Harmand, Werner, and Werner in [18], is one of these geometric properties

Copyright 2016 by the Tusi Mathematical Research Group.

Received Dec. 3, 2015; Accepted Dec. 22, 2015.

${ }^{*}$ Corresponding author.

2010 Mathematics Subject Classification. Primary 46G25; Secondary 47H60, 46B04, 47L22.

Keywords. HB-subspaces, homogeneous polynomials, weakly continuous on bounded sets polynomials. 


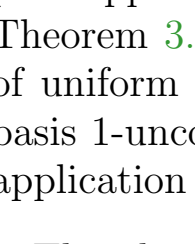

Banach J. Math. Anal. 10 (2016), no. 4, 686-702

http://dx.doi.org/10.1215/17358787-3649854

ISSN: $1735-8787$ (electronic)

http://projecteuclid.org/bjma

ANALYSIS

\title{
IDEAL STRUCTURES IN VECTOR-VALUED POLYNOMIAL SPACES
}

\author{
VERÓNICA DIMANT, ${ }^{1}$ SILVIA LASSALLE, ${ }^{1}$ and ÁNGELES PRIETO ${ }^{2 *}$ \\ Communicated by D. Werner
}

\begin{abstract}
This paper is concerned with the study of geometric structures in spaces of polynomials. More precisely, we discuss for $E$ and $F$ Banach spaces, whether the class of $n$-homogeneous polynomials, $\mathcal{P}_{w}\left({ }^{n} E, F\right)$, which are weakly continuous on bounded sets, is an HB-subspace or an $M(1, C)$-ideal in the space of continuous $n$-homogeneous polynomials, $\mathcal{P}\left({ }^{n} E, F\right)$. We establish sufficient conditions under which the problem can be positively solved. Some examples are given. We also study when some ideal structures pass from $\mathcal{P}_{w}\left({ }^{n} E, F\right)$ as an ideal in $\mathcal{P}\left({ }^{n} E, F\right)$ to the range space $F$ as an ideal in its bidual $F^{* *}$.
\end{abstract}

\section{INTRODUCTION}

Let $X$ be a (real or complex) Banach space, and let $J$ be a closed subspace of $X$. According to the Hahn-Banach theorem, every continuous linear functional $g \in J^{*}$ has an extension $f \in X^{*}$ with the same norm. A long-standing problem is to determine when every functional on $J$ has a unique norm-preserving extension to $X$. This question is closely related to geometric properties of both spaces which, in many cases, imply the existence of a norm 1 projection on $X^{*}$ whose kernel is $J^{\perp}:=\left\{x^{*} \in X^{*}: x^{*}(y)=0\right.$, for all $\left.y \in J\right\}$, the annihilator of $J$. When there exists such a projection, $J$ is said to be an ideal in $X$. A canonical example of this fact is that $X$ is always an ideal in its bidual $X^{* *}$.

The notion of $M$-ideal, introduced by Alfsen and Effros and widely studied by Harmand, Werner, and Werner in [18], is one of these geometric properties

Copyright 2016 by the Tusi Mathematical Research Group.

Received Dec. 3, 2015; Accepted Dec. 22, 2015.

${ }^{*}$ Corresponding author.

2010 Mathematics Subject Classification. Primary 46G25; Secondary 47H60, 46B04, 47L22.

Keywords. HB-subspaces, homogeneous polynomials, weakly continuous on bounded sets polynomials. 


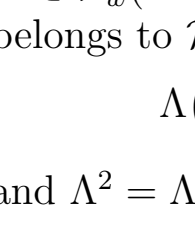

Banach J. Math. Anal. 10 (2016), no. 4, 686-702

http://dx.doi.org/10.1215/17358787-3649854

ISSN: $1735-8787$ (electronic)

http://projecteuclid.org/bjma

ANALYSIS

\title{
IDEAL STRUCTURES IN VECTOR-VALUED POLYNOMIAL SPACES
}

\author{
VERÓNICA DIMANT, ${ }^{1}$ SILVIA LASSALLE, ${ }^{1}$ and ÁNGELES PRIETO ${ }^{2 *}$ \\ Communicated by D. Werner
}

\begin{abstract}
This paper is concerned with the study of geometric structures in spaces of polynomials. More precisely, we discuss for $E$ and $F$ Banach spaces, whether the class of $n$-homogeneous polynomials, $\mathcal{P}_{w}\left({ }^{n} E, F\right)$, which are weakly continuous on bounded sets, is an HB-subspace or an $M(1, C)$-ideal in the space of continuous $n$-homogeneous polynomials, $\mathcal{P}\left({ }^{n} E, F\right)$. We establish sufficient conditions under which the problem can be positively solved. Some examples are given. We also study when some ideal structures pass from $\mathcal{P}_{w}\left({ }^{n} E, F\right)$ as an ideal in $\mathcal{P}\left({ }^{n} E, F\right)$ to the range space $F$ as an ideal in its bidual $F^{* *}$.
\end{abstract}

\section{INTRODUCTION}

Let $X$ be a (real or complex) Banach space, and let $J$ be a closed subspace of $X$. According to the Hahn-Banach theorem, every continuous linear functional $g \in J^{*}$ has an extension $f \in X^{*}$ with the same norm. A long-standing problem is to determine when every functional on $J$ has a unique norm-preserving extension to $X$. This question is closely related to geometric properties of both spaces which, in many cases, imply the existence of a norm 1 projection on $X^{*}$ whose kernel is $J^{\perp}:=\left\{x^{*} \in X^{*}: x^{*}(y)=0\right.$, for all $\left.y \in J\right\}$, the annihilator of $J$. When there exists such a projection, $J$ is said to be an ideal in $X$. A canonical example of this fact is that $X$ is always an ideal in its bidual $X^{* *}$.

The notion of $M$-ideal, introduced by Alfsen and Effros and widely studied by Harmand, Werner, and Werner in [18], is one of these geometric properties

Copyright 2016 by the Tusi Mathematical Research Group.

Received Dec. 3, 2015; Accepted Dec. 22, 2015.

${ }^{*}$ Corresponding author.

2010 Mathematics Subject Classification. Primary 46G25; Secondary 47H60, 46B04, 47L22.

Keywords. HB-subspaces, homogeneous polynomials, weakly continuous on bounded sets polynomials. 


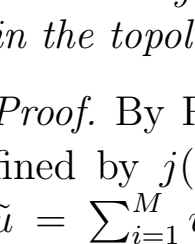

Banach J. Math. Anal. 10 (2016), no. 4, 686-702

http://dx.doi.org/10.1215/17358787-3649854

ISSN: $1735-8787$ (electronic)

http://projecteuclid.org/bjma

ANALYSIS

\title{
IDEAL STRUCTURES IN VECTOR-VALUED POLYNOMIAL SPACES
}

\author{
VERÓNICA DIMANT, ${ }^{1}$ SILVIA LASSALLE, ${ }^{1}$ and ÁNGELES PRIETO ${ }^{2 *}$ \\ Communicated by D. Werner
}

\begin{abstract}
This paper is concerned with the study of geometric structures in spaces of polynomials. More precisely, we discuss for $E$ and $F$ Banach spaces, whether the class of $n$-homogeneous polynomials, $\mathcal{P}_{w}\left({ }^{n} E, F\right)$, which are weakly continuous on bounded sets, is an HB-subspace or an $M(1, C)$-ideal in the space of continuous $n$-homogeneous polynomials, $\mathcal{P}\left({ }^{n} E, F\right)$. We establish sufficient conditions under which the problem can be positively solved. Some examples are given. We also study when some ideal structures pass from $\mathcal{P}_{w}\left({ }^{n} E, F\right)$ as an ideal in $\mathcal{P}\left({ }^{n} E, F\right)$ to the range space $F$ as an ideal in its bidual $F^{* *}$.
\end{abstract}

\section{INTRODUCTION}

Let $X$ be a (real or complex) Banach space, and let $J$ be a closed subspace of $X$. According to the Hahn-Banach theorem, every continuous linear functional $g \in J^{*}$ has an extension $f \in X^{*}$ with the same norm. A long-standing problem is to determine when every functional on $J$ has a unique norm-preserving extension to $X$. This question is closely related to geometric properties of both spaces which, in many cases, imply the existence of a norm 1 projection on $X^{*}$ whose kernel is $J^{\perp}:=\left\{x^{*} \in X^{*}: x^{*}(y)=0\right.$, for all $\left.y \in J\right\}$, the annihilator of $J$. When there exists such a projection, $J$ is said to be an ideal in $X$. A canonical example of this fact is that $X$ is always an ideal in its bidual $X^{* *}$.

The notion of $M$-ideal, introduced by Alfsen and Effros and widely studied by Harmand, Werner, and Werner in [18], is one of these geometric properties

Copyright 2016 by the Tusi Mathematical Research Group.

Received Dec. 3, 2015; Accepted Dec. 22, 2015.

${ }^{*}$ Corresponding author.

2010 Mathematics Subject Classification. Primary 46G25; Secondary 47H60, 46B04, 47L22.

Keywords. HB-subspaces, homogeneous polynomials, weakly continuous on bounded sets polynomials. 


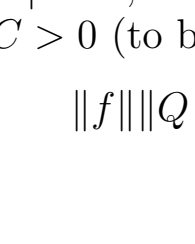

Banach J. Math. Anal. 10 (2016), no. 4, 686-702

http://dx.doi.org/10.1215/17358787-3649854

ISSN: $1735-8787$ (electronic)

http://projecteuclid.org/bjma

ANALYSIS

\title{
IDEAL STRUCTURES IN VECTOR-VALUED POLYNOMIAL SPACES
}

\author{
VERÓNICA DIMANT, ${ }^{1}$ SILVIA LASSALLE, ${ }^{1}$ and ÁNGELES PRIETO ${ }^{2 *}$ \\ Communicated by D. Werner
}

\begin{abstract}
This paper is concerned with the study of geometric structures in spaces of polynomials. More precisely, we discuss for $E$ and $F$ Banach spaces, whether the class of $n$-homogeneous polynomials, $\mathcal{P}_{w}\left({ }^{n} E, F\right)$, which are weakly continuous on bounded sets, is an HB-subspace or an $M(1, C)$-ideal in the space of continuous $n$-homogeneous polynomials, $\mathcal{P}\left({ }^{n} E, F\right)$. We establish sufficient conditions under which the problem can be positively solved. Some examples are given. We also study when some ideal structures pass from $\mathcal{P}_{w}\left({ }^{n} E, F\right)$ as an ideal in $\mathcal{P}\left({ }^{n} E, F\right)$ to the range space $F$ as an ideal in its bidual $F^{* *}$.
\end{abstract}

\section{INTRODUCTION}

Let $X$ be a (real or complex) Banach space, and let $J$ be a closed subspace of $X$. According to the Hahn-Banach theorem, every continuous linear functional $g \in J^{*}$ has an extension $f \in X^{*}$ with the same norm. A long-standing problem is to determine when every functional on $J$ has a unique norm-preserving extension to $X$. This question is closely related to geometric properties of both spaces which, in many cases, imply the existence of a norm 1 projection on $X^{*}$ whose kernel is $J^{\perp}:=\left\{x^{*} \in X^{*}: x^{*}(y)=0\right.$, for all $\left.y \in J\right\}$, the annihilator of $J$. When there exists such a projection, $J$ is said to be an ideal in $X$. A canonical example of this fact is that $X$ is always an ideal in its bidual $X^{* *}$.

The notion of $M$-ideal, introduced by Alfsen and Effros and widely studied by Harmand, Werner, and Werner in [18], is one of these geometric properties

Copyright 2016 by the Tusi Mathematical Research Group.

Received Dec. 3, 2015; Accepted Dec. 22, 2015.

${ }^{*}$ Corresponding author.

2010 Mathematics Subject Classification. Primary 46G25; Secondary 47H60, 46B04, 47L22.

Keywords. HB-subspaces, homogeneous polynomials, weakly continuous on bounded sets polynomials. 


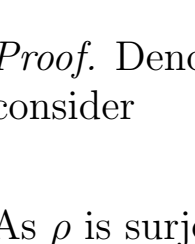

Banach J. Math. Anal. 10 (2016), no. 4, 686-702

http://dx.doi.org/10.1215/17358787-3649854

ISSN: $1735-8787$ (electronic)

http://projecteuclid.org/bjma

ANALYSIS

\title{
IDEAL STRUCTURES IN VECTOR-VALUED POLYNOMIAL SPACES
}

\author{
VERÓNICA DIMANT, ${ }^{1}$ SILVIA LASSALLE, ${ }^{1}$ and ÁNGELES PRIETO ${ }^{2 *}$ \\ Communicated by D. Werner
}

\begin{abstract}
This paper is concerned with the study of geometric structures in spaces of polynomials. More precisely, we discuss for $E$ and $F$ Banach spaces, whether the class of $n$-homogeneous polynomials, $\mathcal{P}_{w}\left({ }^{n} E, F\right)$, which are weakly continuous on bounded sets, is an HB-subspace or an $M(1, C)$-ideal in the space of continuous $n$-homogeneous polynomials, $\mathcal{P}\left({ }^{n} E, F\right)$. We establish sufficient conditions under which the problem can be positively solved. Some examples are given. We also study when some ideal structures pass from $\mathcal{P}_{w}\left({ }^{n} E, F\right)$ as an ideal in $\mathcal{P}\left({ }^{n} E, F\right)$ to the range space $F$ as an ideal in its bidual $F^{* *}$.
\end{abstract}

\section{INTRODUCTION}

Let $X$ be a (real or complex) Banach space, and let $J$ be a closed subspace of $X$. According to the Hahn-Banach theorem, every continuous linear functional $g \in J^{*}$ has an extension $f \in X^{*}$ with the same norm. A long-standing problem is to determine when every functional on $J$ has a unique norm-preserving extension to $X$. This question is closely related to geometric properties of both spaces which, in many cases, imply the existence of a norm 1 projection on $X^{*}$ whose kernel is $J^{\perp}:=\left\{x^{*} \in X^{*}: x^{*}(y)=0\right.$, for all $\left.y \in J\right\}$, the annihilator of $J$. When there exists such a projection, $J$ is said to be an ideal in $X$. A canonical example of this fact is that $X$ is always an ideal in its bidual $X^{* *}$.

The notion of $M$-ideal, introduced by Alfsen and Effros and widely studied by Harmand, Werner, and Werner in [18], is one of these geometric properties

Copyright 2016 by the Tusi Mathematical Research Group.

Received Dec. 3, 2015; Accepted Dec. 22, 2015.

${ }^{*}$ Corresponding author.

2010 Mathematics Subject Classification. Primary 46G25; Secondary 47H60, 46B04, 47L22.

Keywords. HB-subspaces, homogeneous polynomials, weakly continuous on bounded sets polynomials. 


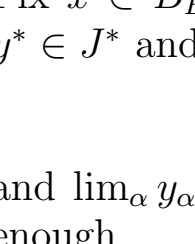

Banach J. Math. Anal. 10 (2016), no. 4, 686-702

http://dx.doi.org/10.1215/17358787-3649854

ISSN: $1735-8787$ (electronic)

http://projecteuclid.org/bjma

ANALYSIS

\title{
IDEAL STRUCTURES IN VECTOR-VALUED POLYNOMIAL SPACES
}

\author{
VERÓNICA DIMANT, ${ }^{1}$ SILVIA LASSALLE, ${ }^{1}$ and ÁNGELES PRIETO ${ }^{2 *}$ \\ Communicated by D. Werner
}

\begin{abstract}
This paper is concerned with the study of geometric structures in spaces of polynomials. More precisely, we discuss for $E$ and $F$ Banach spaces, whether the class of $n$-homogeneous polynomials, $\mathcal{P}_{w}\left({ }^{n} E, F\right)$, which are weakly continuous on bounded sets, is an HB-subspace or an $M(1, C)$-ideal in the space of continuous $n$-homogeneous polynomials, $\mathcal{P}\left({ }^{n} E, F\right)$. We establish sufficient conditions under which the problem can be positively solved. Some examples are given. We also study when some ideal structures pass from $\mathcal{P}_{w}\left({ }^{n} E, F\right)$ as an ideal in $\mathcal{P}\left({ }^{n} E, F\right)$ to the range space $F$ as an ideal in its bidual $F^{* *}$.
\end{abstract}

\section{INTRODUCTION}

Let $X$ be a (real or complex) Banach space, and let $J$ be a closed subspace of $X$. According to the Hahn-Banach theorem, every continuous linear functional $g \in J^{*}$ has an extension $f \in X^{*}$ with the same norm. A long-standing problem is to determine when every functional on $J$ has a unique norm-preserving extension to $X$. This question is closely related to geometric properties of both spaces which, in many cases, imply the existence of a norm 1 projection on $X^{*}$ whose kernel is $J^{\perp}:=\left\{x^{*} \in X^{*}: x^{*}(y)=0\right.$, for all $\left.y \in J\right\}$, the annihilator of $J$. When there exists such a projection, $J$ is said to be an ideal in $X$. A canonical example of this fact is that $X$ is always an ideal in its bidual $X^{* *}$.

The notion of $M$-ideal, introduced by Alfsen and Effros and widely studied by Harmand, Werner, and Werner in [18], is one of these geometric properties

Copyright 2016 by the Tusi Mathematical Research Group.

Received Dec. 3, 2015; Accepted Dec. 22, 2015.

${ }^{*}$ Corresponding author.

2010 Mathematics Subject Classification. Primary 46G25; Secondary 47H60, 46B04, 47L22.

Keywords. HB-subspaces, homogeneous polynomials, weakly continuous on bounded sets polynomials. 


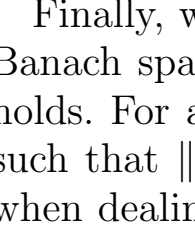

Banach J. Math. Anal. 10 (2016), no. 4, 686-702

http://dx.doi.org/10.1215/17358787-3649854

ISSN: $1735-8787$ (electronic)

http://projecteuclid.org/bjma

ANALYSIS

\title{
IDEAL STRUCTURES IN VECTOR-VALUED POLYNOMIAL SPACES
}

\author{
VERÓNICA DIMANT, ${ }^{1}$ SILVIA LASSALLE, ${ }^{1}$ and ÁNGELES PRIETO ${ }^{2 *}$ \\ Communicated by D. Werner
}

\begin{abstract}
This paper is concerned with the study of geometric structures in spaces of polynomials. More precisely, we discuss for $E$ and $F$ Banach spaces, whether the class of $n$-homogeneous polynomials, $\mathcal{P}_{w}\left({ }^{n} E, F\right)$, which are weakly continuous on bounded sets, is an HB-subspace or an $M(1, C)$-ideal in the space of continuous $n$-homogeneous polynomials, $\mathcal{P}\left({ }^{n} E, F\right)$. We establish sufficient conditions under which the problem can be positively solved. Some examples are given. We also study when some ideal structures pass from $\mathcal{P}_{w}\left({ }^{n} E, F\right)$ as an ideal in $\mathcal{P}\left({ }^{n} E, F\right)$ to the range space $F$ as an ideal in its bidual $F^{* *}$.
\end{abstract}

\section{INTRODUCTION}

Let $X$ be a (real or complex) Banach space, and let $J$ be a closed subspace of $X$. According to the Hahn-Banach theorem, every continuous linear functional $g \in J^{*}$ has an extension $f \in X^{*}$ with the same norm. A long-standing problem is to determine when every functional on $J$ has a unique norm-preserving extension to $X$. This question is closely related to geometric properties of both spaces which, in many cases, imply the existence of a norm 1 projection on $X^{*}$ whose kernel is $J^{\perp}:=\left\{x^{*} \in X^{*}: x^{*}(y)=0\right.$, for all $\left.y \in J\right\}$, the annihilator of $J$. When there exists such a projection, $J$ is said to be an ideal in $X$. A canonical example of this fact is that $X$ is always an ideal in its bidual $X^{* *}$.

The notion of $M$-ideal, introduced by Alfsen and Effros and widely studied by Harmand, Werner, and Werner in [18], is one of these geometric properties

Copyright 2016 by the Tusi Mathematical Research Group.

Received Dec. 3, 2015; Accepted Dec. 22, 2015.

${ }^{*}$ Corresponding author.

2010 Mathematics Subject Classification. Primary 46G25; Secondary 47H60, 46B04, 47L22.

Keywords. HB-subspaces, homogeneous polynomials, weakly continuous on bounded sets polynomials. 


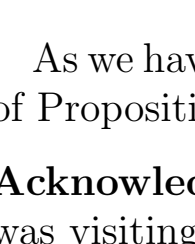

Banach J. Math. Anal. 10 (2016), no. 4, 686-702

http://dx.doi.org/10.1215/17358787-3649854

ISSN: $1735-8787$ (electronic)

http://projecteuclid.org/bjma

ANALYSIS

\title{
IDEAL STRUCTURES IN VECTOR-VALUED POLYNOMIAL SPACES
}

\author{
VERÓNICA DIMANT, ${ }^{1}$ SILVIA LASSALLE, ${ }^{1}$ and ÁNGELES PRIETO ${ }^{2 *}$ \\ Communicated by D. Werner
}

\begin{abstract}
This paper is concerned with the study of geometric structures in spaces of polynomials. More precisely, we discuss for $E$ and $F$ Banach spaces, whether the class of $n$-homogeneous polynomials, $\mathcal{P}_{w}\left({ }^{n} E, F\right)$, which are weakly continuous on bounded sets, is an HB-subspace or an $M(1, C)$-ideal in the space of continuous $n$-homogeneous polynomials, $\mathcal{P}\left({ }^{n} E, F\right)$. We establish sufficient conditions under which the problem can be positively solved. Some examples are given. We also study when some ideal structures pass from $\mathcal{P}_{w}\left({ }^{n} E, F\right)$ as an ideal in $\mathcal{P}\left({ }^{n} E, F\right)$ to the range space $F$ as an ideal in its bidual $F^{* *}$.
\end{abstract}

\section{INTRODUCTION}

Let $X$ be a (real or complex) Banach space, and let $J$ be a closed subspace of $X$. According to the Hahn-Banach theorem, every continuous linear functional $g \in J^{*}$ has an extension $f \in X^{*}$ with the same norm. A long-standing problem is to determine when every functional on $J$ has a unique norm-preserving extension to $X$. This question is closely related to geometric properties of both spaces which, in many cases, imply the existence of a norm 1 projection on $X^{*}$ whose kernel is $J^{\perp}:=\left\{x^{*} \in X^{*}: x^{*}(y)=0\right.$, for all $\left.y \in J\right\}$, the annihilator of $J$. When there exists such a projection, $J$ is said to be an ideal in $X$. A canonical example of this fact is that $X$ is always an ideal in its bidual $X^{* *}$.

The notion of $M$-ideal, introduced by Alfsen and Effros and widely studied by Harmand, Werner, and Werner in [18], is one of these geometric properties

Copyright 2016 by the Tusi Mathematical Research Group.

Received Dec. 3, 2015; Accepted Dec. 22, 2015.

${ }^{*}$ Corresponding author.

2010 Mathematics Subject Classification. Primary 46G25; Secondary 47H60, 46B04, 47L22.

Keywords. HB-subspaces, homogeneous polynomials, weakly continuous on bounded sets polynomials. 


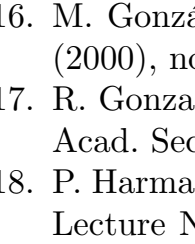

Banach J. Math. Anal. 10 (2016), no. 4, 686-702

http://dx.doi.org/10.1215/17358787-3649854

ISSN: $1735-8787$ (electronic)

http://projecteuclid.org/bjma

ANALYSIS

\title{
IDEAL STRUCTURES IN VECTOR-VALUED POLYNOMIAL SPACES
}

\author{
VERÓNICA DIMANT, ${ }^{1}$ SILVIA LASSALLE, ${ }^{1}$ and ÁNGELES PRIETO ${ }^{2 *}$ \\ Communicated by D. Werner
}

\begin{abstract}
This paper is concerned with the study of geometric structures in spaces of polynomials. More precisely, we discuss for $E$ and $F$ Banach spaces, whether the class of $n$-homogeneous polynomials, $\mathcal{P}_{w}\left({ }^{n} E, F\right)$, which are weakly continuous on bounded sets, is an HB-subspace or an $M(1, C)$-ideal in the space of continuous $n$-homogeneous polynomials, $\mathcal{P}\left({ }^{n} E, F\right)$. We establish sufficient conditions under which the problem can be positively solved. Some examples are given. We also study when some ideal structures pass from $\mathcal{P}_{w}\left({ }^{n} E, F\right)$ as an ideal in $\mathcal{P}\left({ }^{n} E, F\right)$ to the range space $F$ as an ideal in its bidual $F^{* *}$.
\end{abstract}

\section{INTRODUCTION}

Let $X$ be a (real or complex) Banach space, and let $J$ be a closed subspace of $X$. According to the Hahn-Banach theorem, every continuous linear functional $g \in J^{*}$ has an extension $f \in X^{*}$ with the same norm. A long-standing problem is to determine when every functional on $J$ has a unique norm-preserving extension to $X$. This question is closely related to geometric properties of both spaces which, in many cases, imply the existence of a norm 1 projection on $X^{*}$ whose kernel is $J^{\perp}:=\left\{x^{*} \in X^{*}: x^{*}(y)=0\right.$, for all $\left.y \in J\right\}$, the annihilator of $J$. When there exists such a projection, $J$ is said to be an ideal in $X$. A canonical example of this fact is that $X$ is always an ideal in its bidual $X^{* *}$.

The notion of $M$-ideal, introduced by Alfsen and Effros and widely studied by Harmand, Werner, and Werner in [18], is one of these geometric properties

Copyright 2016 by the Tusi Mathematical Research Group.

Received Dec. 3, 2015; Accepted Dec. 22, 2015.

${ }^{*}$ Corresponding author.

2010 Mathematics Subject Classification. Primary 46G25; Secondary 47H60, 46B04, 47L22.

Keywords. HB-subspaces, homogeneous polynomials, weakly continuous on bounded sets polynomials. 